\title{
Non-Destructive Imaging and Spectroscopy Methods using Laser Light
}

\author{
Hubert Grün ${ }^{1}$, Bernhard Reitinger ${ }^{1}$, Andreas Buchsbaum ${ }^{1}$, Stefan Katletz ${ }^{1}$ \\ ${ }^{1}$ RECENDT Research Center for Non-Destructive Testing GmbH, Altenberger Str. 69, 4040 Linz, \\ Austria \\ hubert.gruen@recendt.at
}

\begin{abstract}
Plenty of optical measurement technologies use lasers. Three technologies using a laser in different ways to acquire various parameters are presented in this work. In Optical Coherence Tomography (OCT) the photons are utilized for imaging. In OCT photons which are backscattered at interfaces within the sample can be measured by using a white light interferometer. This provides a good axial resolution but in general, OCT is limited only to a little imaging depth because of the need of ballistic, non-scattered photons. In laser ultrasound technology short laser pulses are used to generate ultrasonic waves without any physical contact. Short laser pulses are absorbed at the surface and thereby broadband ultrasonic signals launched. The wave propagates through the sample and is reflected and backscattered on interfaces. A laser vibrometer is used for non-contact measurement of the ultrasonic waves arriving at the sample surface. Terahertz imaging and spectroscopy uses a laser to produce the waves necessary for imaging. Laser pulses in the range of femto seconds excite terahertz pulses in a photoconductive antenna. During the propagation of the terahertz radiation trough the sample under investigation the waves experience attenuation and retardation which can be detected with an additional photoconductive antenna. Besides imaging, the terahertz pulse also can be Fourier transformed for spectroscopic applications. All these non-destructive testing methods uses laser light to gain different information at different imaging depth and spatial resolutions for different materials and applications.
\end{abstract}

Key words: non-destructive imaging, optical sensor, optical coherence tomography, laser ultrasound, terahertz imaging and spectroscopy

\section{Introduction}

In the field of non-destructive testing a multitude of different technologies are used to detect any kinds of faults and deviations. Especially optical measurement technologies are applied besides detectors and technologies based on other physical principles. Several of these methods incorporate a pulsed or continuous wave laser at varying wavelength.

Three emerging technologies using lasers as a centerpiece are presented in this work. In these NDT techniques the laser light fulfils different functions. In Optical Coherence Tomography (OCT) the continuous wave laser light in the near infrared is directly used for imaging. The interaction of ballistic photons with matter causes back reflection that is measured. Laser Ultrasound (LUS) uses short laser pulses (in the range from pico seconds to nano seconds) which are absorbed at the surface of the sample. Thereby ultrasonic waves are excited due to thermoelastic expansion or ablation - depending on the pulse energy. The contactless created broadband ultrasonic waves propagate through the sample and thereby the imaging contrast is created by the interaction of the ultrasonic waves with matter. In LUS the wavelength of the excitation laser ranges from visible light to the mid infrared depending on the absorption capabilities of the sample. On the other hand one can use laser pulses which are shorter than in LUS. Terahertz imaging and spectroscopy involves femto second laser pulses in the near infrared wavelength. The pulses interact with a special semiconductor and thereby terahertz radiation (T-rays) is launched. These terahertz waves can be used for imaging and spectroscopy due to attenuation and retardation of the waves while interacting with matter. Three different kinds of lasers are serving as source for diverse kinds of waves: ultrasonic waves and electromagnetic waves in two totally different wavelength regions. This way each technology leads to particular information content of the measured signal since the interaction of the waves with matter is different. 
In the following paragraphs the three different techniques Optical Coherence Tomography, Laser Ultrasound and Terahertz imaging and spectroscopy are explained in detail and some examples of applications are given.

\section{Optical Coherence Tomography}

Optical coherence tomography (OCT) [1] is an emerging non-destructive and contactless imaging technique, which allows real-time and in situ acquisition of high resolution one-, two-, and three-dimensional image data. OCT is based on the physical phenomenon of white light interferometry and therefore well suited to image layered structures with thickness in the range of few microns. The image contrast is due to inhomogeneities in the refractive index of the sample materials. Thus OCT provides complementary information to other high resolution imaging techniques like ultrasound, $\mathrm{X}$ ray tomography (CT) and magnetic resonance imaging (MRI).

The simplest OCT approach includes a modified Michelson interferometer, where the mirror in one of the interferometer arms is replaced by the sample. The probing beam is focused into the object and photons are back-scattered from structures like interfaces, impurities, pores, or cells at different depths of the sample. By comparing the arrival times of the scattered photons with a reference light beam a depth scan is obtained. With the aid of galvanometer mirrors the probing beam is scanned laterally across the sample. By subsequent acquisition of depth scans at adjacent lateral positions a depth resolved cross section (2D image) can be reconstructed. Several bordering cross sections can be stick together resulting in a volume (3D image).

The depth resolved OCT signal can be acquired either in the time- or frequency-domain, with the latter approach offering advantages in terms of imaging speed and sensitivity [2], enabling video rate imaging [3] and in-line applications. In timedomain OCT the length of the reference arm in the interferometer is scanned over several millimeters while the sample is kept in place. Due to the interferometric detection scheme and the short coherence length of the used light sources, a signal is detected only if the photons reflected from both interferometer arms have travelled the same optical distance to the photodetector. Otherwise only noise is detected. The mechanical movement of the reference mirror is rather time consuming and may lead to mechanical instabilities and noise. One way to speed up the image acquisition process is to use the so-called Fourier-domain OCT approach. Here the reference mirror is fixed and the light coming from both reference and object is detected in a spectrally resolved way. This can either be done in parallel (spectral-domain OCT) by using a dispersing element and a chargecoupled-device (CCD) or Complementary Metal Oxide Semiconductor (CMOS) camera, or sequentially by scanning a narrow laser line across a broad spectral region (swept-source OCT). The schematic of a typical spectraldomain OCT setup is depicted in Figure 1. In Fourier-domain OCT the depth information is encoded in a cosinusoidal modulation of the acquired spectrum and can be accessed by applying an inverse Fourier transform. With state-of-the-art cameras or swept laser sources frame rates of several hundred $\mathrm{kHz}$ can be achieved.

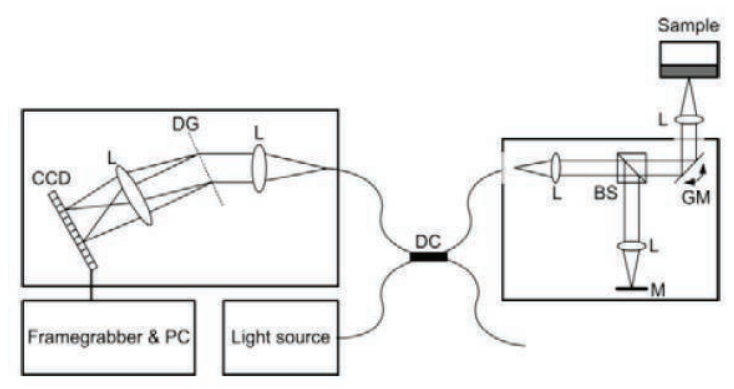

Fig. 1. Schematic of a spectral-domain OCT system. The dashed boxes represent portable and independent modules. DC - directional coupler; BS beamsplitter; (G)M - (galvanometer) mirror; $L$ - lens; $D G$ - diffraction grating; $C C D$ - Charge-coupled device camera.

One particularity of OCT is the fact that the lateral (transversal) and axial (depth) resolutions are decoupled from each other. The former is determined by the applied wavelength and the numerical aperture of the focusing optics in the object arm. A high numerical aperture leads to a small focal spot size and therefore to a high lateral resolution. Sharp focusing, however, not only results in a small spot size in the lateral dimension, but also in a reduced depth of field in the axial dimension [2]. Using a low numerical aperture objective leads to a large depth of field at the expense of a decreased lateral resolution. The axial resolution, on the other hand, is limited by the spectral characteristics of the used light source and depends on the square of the center wavelength and the inverse of the spectral bandwidth. With advanced light sources axial resolutions below one micron can be achieved.

So far, the research in the field of OCT has mainly been directed towards biomedical applications like ophthalmology [1], dermatology [4], dentistry [5], or development biology [6]. However, over the last years, the number of OCT applications outside the field of biomedicine has increased substantially [7-11]. 
OCT can provide both quantitative and qualitative information. The former includes e.g. the thickness of layers, or the size and distribution of pores or cells. With the aid of state-of-the-art image processing tools it is possible to automatically analyze sample features and to monitor structural changes over time. Qualitative information is obtained through the visual inspection of the acquired images. Features like surfaces, impurities or cells can be detected and give rapid indications on the structural condition of the analyzed device, providing important information to the technician. Besides providing images in which the contrast is based on different reflectivities, OCT also allows the study of sample characteristics like the attenuation properties (e.g. differential absorption OCT; DA-OCT) [12], the flow velocity and direction of fluids (Doppler OCT) [13], and birefringence due to strain or anisotropies (polarization sensitive OCT; PSOCT) [14]. With such functional approaches it is possible to monitor changes in the chemical composition, to detect congestions in tubes, or to analyze the effect of cooling or heating processes on the sample.

\section{Laser Ultrasound}

Laser Ultrasound is a contactless nondestructive ultrasound testing method. The ultrasonic waves are generated by thermoelastic expansion or ablation of the surface by irradiation of a short laser pulse [15]. Thus the sample itself is the ultrasonic generator and determines the direction of propagation of the sound waves. The absorption of the laser pulse leads to both, bulk waves (longitudinal and transversal waves) and surface acoustic waves (Rayleigh waves, Lamb waves, Scholte waves...) which propagate through the sample (see Fig. 2). The detection of the ultrasonic wave is done also contactless with a laser vibrometer (mostly homodyne interferometers like confocalFabry-Perot or TWM two wave mixing interferometers) $[16,17]$ which measures the displacement of the surface caused by the ultrasonic waves. A laser beam is reflected from the surface of the sample under investigation and gets phase modulated (Doppler shifted) by the vibrations. The phase modulation is further demodulated in amplitude modulations by an interferometer and is therefore easy to detect via photodetectors which are able to operate up to very high frequencies.

This results in several advantages over the conventional ultrasound. First of all the contactless generation and detection of ultrasonic waves. For instance the distance of the laser ultrasonic detection head to the sample surface can be very large (up to one meter) with a focusing tolerance in the centimeter region making it very insensitive to sample movements (compared to air coupled ultrasound, EMAT electromagnetic acoustic transducers and eddycurrent testing methods).

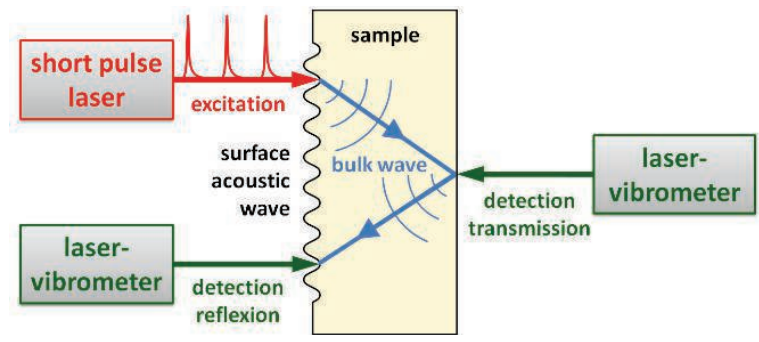

Fig. 2. Schematic of the laser ultrasound principle.

The direction of the ultrasonic wave is not determined by the angle of the incident excitation laser. The sample itself is the ultrasound generator and thus determines the direction of ultrasonic waves which is perpendicular to the sample surface.

Laser Ultrasound provides high lateral resolution by the ability to focus the generation and detection beams to small spots $(100 \mu \mathrm{m}$ diameter), enabling the testing on complicated structures like tight curves and small areas like on tooth flanks of a gearwheel or a spindle. In general the resolution strongly depends on the material to be characterized.

The advantages of laser ultrasound like contactless, non-destructive, high possible distances between measurement head and sample and particularly the generation and detection of high ultrasound frequencies (with a broad bandwidth) lead to remarkable applications. One of the first industrial applications for laser ultrasound was the detection of the thickness of tubes during production, when they are extremely hot $\left(>1000^{\circ} \mathrm{C}\right)$. This was started in the nineties by the group of J.P. Monchalin (IMI - Montreal) and industrially implemented by a company named Tecnar. Further defect detection of CFRP components like delaminations and characterization of porosities were started by Lockheed Martin to support their development of new materials on military planes. Further applications are the defect detection in welds like cracks and pores, the characterization of grain structure in metals during heating and several other applications which are also possible to be examined with conventional ultrasound.

\section{Terahertz Imaging and Spectroscopy}

Terahertz (THz) imaging is a non-destructive and contactless imaging technique, which allows acquisition of two-, and three-dimensional image data. With terahertz waves it is also possible to 
perform spectroscopy. Terahertz waves, loosely defined as the spectral range covering $3 \mathrm{~mm}$ to $30 \mu \mathrm{m}(0.1-10 \mathrm{THz})$, exhibit very low absorption in many dielectric materials. The resulting large penetration depth (several millimeters to centimeters) can therefore be used for many non-destructive measurement tasks. Another advantage is that a typical time-domain (TD) setup (see Fig. 3) measures the time-resolved transmitted or reflected $\mathrm{THz}$ pulse (phasesensitive), i.e., the technique is depth sensitive. For the generation of a $\mathrm{THz}$ pulse, a very short femtosecond pulse from a titanium:sapphire laser is focused onto a $\mathrm{THz}$ emitter antenna made from low-temperature grown gallium arsenide. This antenna then emits an electromagnetic pulse of a few picoseconds length, which can be collimated and focused by off-axis parabolic mirrors or polymers with very low attenuation (e.g. PE, PP, TPX). A second antenna is used as a detector, which needs to be illuminated by another femtosecond pulse from the laser, but which can be delayed by a mechanical translation stage (pump-and-probe technique).

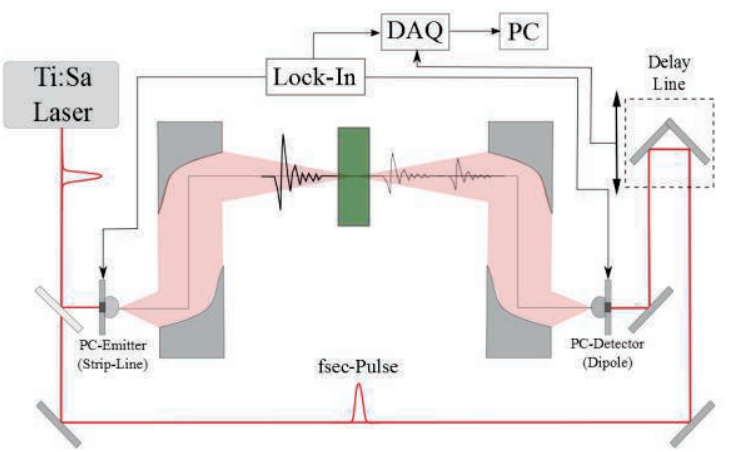

Fig. 3. Typical THz TD setup for transmission measurements. [18]

The time axis is generated by the spatial displacement of the mechanical delay line. This time resolved pulse also contains spectral information, which can be obtained by a Fourier transformation of the time signal. Especially carbohydrate chains (like sugars), polymers, pharmaceuticals, petrochemicals, plastic explosives, etc. can be detected by characteristic absorption lines in the $\mathrm{THz}$ region. In addition, also the refractive index can be determined directly from the measurement data, which also allows to determine, e.g., the density of plastics (LDPE, HDPE, UHMWPE) [19]. This can also be used to monitor the curing of, e.g. dental composites [20].

In order to create images, the sample is raster scanned, i.e. in every point a complete spectrum is recorded. Alternatively, also the $\mathrm{THz}$ beam can be deflected and thereby scans an object [21].
The depth resolved measurement capability shows the interfaces of a multilayered polymer tube. This image was obtained in reflection, i.e., the technique could be potentially used for in-line monitoring of the thickness of the individual layers during the co-extrusion of the pipe.

Finally these measurements can also be carried out polarization sensitive, which then allows to determine the birefringence and orientation of the optical axis is anisotropic materials [18,22]. Furthermore this permits to contact-less measurements of the mechanical stress in elastomers [23].

\section{Conclusion and Outlook}

Three optical measurement technologies for non-destructive testing were presented. These methods use lasers for gaining information of the samples with a spatial resolution ranging from a few microns (OCT) to sub-millimeter range ( $\mathrm{THz}$ ) and LUS in between. Due to the unequal nature of the different waves created with the three technologies, complementary information can be obtained. OCT yields information about the optical properties, LUS measures acoustical parameters and $\mathrm{THz}$ collects information about the interaction of T-rays with matter. Due to the different wavelength the penetration depth varies - depending on the material - from a few millimeters (OCT) to several centimeters ( $\mathrm{THz}$ ). All the presented technologies are applicable in industrial environment as first experiments showed. Due to further progress in terms of measurement speed and robustness and a drop in hardware cost more and more industrial applications become interesting for these techniques.

\section{Acknowledgement}

We acknowledge financial support from the European Regional Development Fund (EFRE) in the framework of the EU-program REGIO 13, and the Federal State of Upper Austria.

\section{References}

[1] D. Huang, et.al, Optical Coherence Tomography, Science 254, 1178-1181 (1991); doi: 10.1126/science.1957169

[2] R. Leitgeb, C. Hitzenberger, and A. Fercher, Performance of fourier domain vs. time domain optical coherence tomography, Opt. Express 11, 889-894 (2003); doi: 10.1364/OE.11.000889

[3] S. van der Jeught, A. Bradu, and A. Podoleanu, Real-time resampling in Fourier domain optical coherence tomography using a graphics processing board, Journal of Biomedical Optics 15(3), 030511 (2010); doi: 10.1117/1.3437078

[4] A. Podoleanu, J. Rogers, D. Jackson, and S. Dunne, Three dimensional OCT images from 
retina and skin, Optics Express 7(9), 292-298 (2000); doi: 10.1364/OE.7.000292

[5] B. Colston, U. Sathyam, L. DaSilva, M. Everett, P. Stroeve, and L. Otis, Dental OCT, Optics Express 3(6), 230-238 (1998); doi: 10.1364/OE.3.000230

[6] A. Davis, S.A. Boppart, F. Rothenberg, and J. Izatt, OCT Applications in Developmental Biology, Drexler W, Fujimoto JG, editors. Optical Coherence Tomography: Technology and Applications, Berlin: Springer. p $919-960$ (2007); doi: 10.1007/978-3-540-77550-8_30

[7] H. Liang, M.G. Cid, R.G. Cucu, G.M. Dobre, A.G. Podoleanu, J. Pedro, and D. Saunders, En-face optical coherence tomography - a novel application of non-invasive imaging to art conservation, Optics Express 13(16), 6133-6144 (2005); doi: 10.1364/OPEX.13.006133

[8] K. Wiesauer, et.al, En-face scanning optical coherence tomography with ultra-high resolution for material investigation, Optics Express 13(3), 1015-1024 (2005); doi: 10.1364/OPEX.13.001015

[9] D. Stifter, Beyond biomedicine: a review of alternative applications and developments for optical coherence tomography, Applied Physics $B$ : Lasers and Optics 88(3), 337-357 (2007); doi: $10.1007 / \mathrm{s} 00340-007-2743-2$

[10] K. Wiesauer, M. Pircher, E. Götzinger, C.K. Hitzenberger, R. Oster, D. Stifter, Investigation of glass-fibre reinforced polymers by polarisationsensitive, ultra-high resolution optical coherence tomography: Internal structures, defects and stress, Composites Science and Technology 67(15-16), 3051-3058 (2007); doi: 10.1016/j.compscitech.2007.04.018

[11] P. Targowski, M. Iwanicka, L. TyminlaskaWidmer, M. Sylwestrzak, E.A. Kwiatkowska, Structural Examination of Easel Paintings with Optical Coherence Tomography, Accounts of Chemical Research 43(6), 826-836 (2009); doi: 10.1021/ar900195d

[12] M. Pircher, E. Götzinger, R. Leitgeb, A. Fercher, and $\mathrm{C}$. Hitzenberger, Measurement and imaging of water concentration in human cornea with differential absorption optical coherence tomography, Opt. Express 11, 2190-2197 (2003); doi: 10.1364/OE.11.002190

[13] Z. Chen, Z. Milner, D. Dave, and J. Nelson J, Optical Doppler tomographic imaging of fluid flow velocity in highly scattering media, Optic Letters 22, 64-66 (1997); doi: 10.1364/OL.22.000064

[14] K. Wiesauer et.al, Transversal ultrahighresolution polarizationsensitive optical coherence tomography for strain mapping in materials, Opt. Express 14, 5945-5953 (2006); doi: 10.1364/OE.14.005945

[15] K.L. Telschow, and R.J. Conant, Optical and thermal parameter effects on laser-generated ultrasound, Journal of the Acoustical Society of
America, Vol. 88, p. 1494 (1990); doi: 10.1121/1.400306

[16] J.P. Monchalin, Optical-Detection of Ultrasound, IEEE Transactions on Ultrasonics, Ferroelectrics and Frequency Control, Vol. 33, p. 485 (1986); doi: 10.1109/T-UFFC.1986.26860

[17] J.P. Monchalin, et al., Laser-Ultrasonics: From the Laboratory to the Shop Floor, Advanced Performance Materials, Vol. 5, pp. 7-23 (1998); doi: 10.1023/A:1008644903553

[18] M. Pfleger, H. Roitner, H. Pühringer, K. Wiesauer, H. Grün, and S. Katletz, Advanced birefringence measurements in standard terahertz time-domain spectroscopy, Appl. Optics 53, pp. 3183-3190 (2014); doi: 10.1364/AO.53.003183

[19] S. Wietzke, C. Jansen, M. Reuter, T. Jung, D. Kraft, S. Chatterjee, B. Fischer, and M. Koch, Terahertz spectroscopy on polymers: A review of morphological studies, J. Mol. Struct., vol. 1006, pp. 41-51 (2011); doi: 10.1016/j.molstruc.2011.07.036

[20] M. Schwerdtfeger, S. Lippert, M. Koch, A. Berg, S. Katletz, and K. Wiesauer, Terahertz timedomain spectroscopy for monitoring the curing of dental composites, Biomed. Opt. Express, vol. 3 (11), pp. 2842-2850 (2012); doi: 10.1364/BOE.3.002842

[21] S. Katletz, M. Pfleger, H. Pühringer, N. Vieweg, B. Scherger, B. Heinen, M. Koch, and K. Wiesauer, Efficient terahertz en-face imaging, Opt. Express 19(23), pp. 23042-23053 (2011); doi: 10.1364/OE.19.023042

[22] S. Katletz, M. Pfleger, H. Pühringer, M. Mikulics, N. Vieweg, O. Peters, B. Scherger, M. Scheller, M. Koch, and K. Wiesauer, Polarization sensitive terahertz imaging: detection of birefringence and optical axis, Opt. Express 20(21), pp. 2302523035 (2012); doi: 10.1364/OE.20.023025

[23] M. Pfleger, H. Pühringer, and S. Katletz, Terahertz-elasticity measurements on elastomers, in 39th International Conference on Infrared, Millimeter and Terahertz Waves (IRMMW-THz), 2014, Tucson, Arizona, pp. 1-2, (2014); doi: 10.1109/IRMMW-THz.2014.6956129 